\title{
Mortality after cancer among patients with diabetes mellitus: effect of diabetes duration and treatment: (questionable) classification of diabetic patients based on combination of specific glucose-lowering drugs
}

\author{
Sarah E. Holden • Christian A. Bannister • \\ Craig J. Currie
}

Received: 2 May 2014 / Accepted: 7 May 2014 / Published online: 3 June 2014

(C) Springer-Verlag Berlin Heidelberg 2014

Keywords Cancer · Diabetes · Diabetes duration · Insulin · Mortality $\cdot$ Treatment

\section{Abbreviation \\ OHA Oral hypoglycaemic agent}

To the Editor: We read the article by Ranc and colleagues with interest [1]; however, we have concerns about its value. This large-scale study investigated the differences in survival following incident cancer in people with and without diabetes, accounting for diabetes treatment and diabetes duration.

Whilst the National Diabetes Register has the advantage of providing nationwide data for the whole of Denmark, the lack of information on specific diabetes treatments and diabetes type is a major limitation of this study. In the study, people with diabetes were classified into three groups: people not receiving any glucose-lowering medication, those prescribed insulin and those prescribed oral hypoglycaemic agents (OHAs) at the time of their cancer diagnosis. It was not clear how people prescribed insulin in combination with other glucose-lowering medicines were classified. Neither was it clear whether OHAs included glucagon-like peptide-1 (GLP-1) agonists. In addition, as the effect of glucoselowering agents on survival in cancer patients was a primary aim, it was surprising that the effects of individual classes of glucose-lowering agents were not investigated individually. The combined OHA group presumably contains several types of glucose-lowering medication with different mechanisms of

S. E. Holden · C. A. Bannister · C. J. Currie $(\bowtie)$

School of Medicine, The Pharma Research Centre, Cardiff

Medicentre, Cardiff University, Cardiff CF14 4UJ, UK

e-mail: currie@cardiff.ac.uk action and therefore different effects on cancer progression. For example, metformin may prevent cancer through the activation of the AMP kinase pathway [2] and, in epidemiological studies, sulfonylurea monotherapy has been associated with an increased risk of cancer relative to metformin (adjusted HR 1.36; 95\% CI 1.19, 1.54) [3]. Patients were classified by diagnosis of diabetes and diabetes treatment type at baseline. It is not clear how changes in treatment during the study period or the subsequent development of diabetes following the index date were accounted for. While the diabetes group treated with no medication and that treated with OHAs are likely to have contained people with type 2 diabetes only, the insulin group is likely to be composed of a combination of patients with type 1 and type 2 diabetes. These patients may well differ in many respects, including age, insulin dose, comorbidity and BMI and therefore differ with respect to the risk of death following cancer diagnosis.

It is difficult to understand the characteristics of the cohort, since their baseline characteristics were not presented. As the authors acknowledge, adjustment for confounders was limited. The Cox model was only adjusted for age, calendar time and diabetes status. Smoking status and comorbidities could not be accounted for. These deficiencies in the data should limit the conclusions that can be drawn from this study. However, in the first paragraph of the discussion, as a primary explanation, the authors postulate that patients treated with OHAs and insulin have a larger degree of comorbidity at the time of cancer diagnosis and therefore a higher rate of mortality. Although this conclusion seems reasonable, it emphasises the limitations of the study, where neither the cause of death nor comorbidities were investigated or accounted for, making it difficult to determine if there was any additional treatment effect. In addition, this suggestion seems to be at odds with the conclusion that diabetes duration did not 
influence prognosis, as it would generally be expected that comorbidity would increase with diabetes duration. Could the grouping of the patients, which essentially defines the position in the diabetes treatment pathway, be acting as a proxy for diabetes duration?

A further analysis including only those people with a duration of diabetes of 2 years at cancer diagnosis was carried out, but the reason for using this arbitrary cutoff was not explained. Reporting the number in each group would also have been useful. In addition, this selection criterion is likely to have had an impact on the characteristics of people included in the insulin group, with the inclusion of more people with type 1 diabetes, latent autoimmune diabetes in adults (LADA) and more advanced type 2 diabetes. In addition, no source of funding or duality of interest was declared.

The patterns of modelled data would be more persuasive if the authors had provided actual survival data to better understand how the unusual biological patterns of survival from their statistical models had been distilled.
Duality of interest CJC is a director of and SEH is employed by Pharmatelligence, a research consultancy receiving funding from pharmaceutical companies. $\mathrm{CAB}$ and $\mathrm{SEH}$ receive fully funded $\mathrm{PhD}$ studentships from Cardiff University. CJC has received funding from and has consulted for various health-related organisations and pharmaceutical manufacturers including pharmaceutical companies that manufacture medicines for the management of diabetes.

Contribution statement SEH wrote the first draft of the letter and CJC and $\mathrm{CAB}$ critically revised this draft. All authors declare that they read and approved the final version of the letter before submission.

\section{References}

1. Ranc K, Jørgensen ME, Friis S, Carstensen B (2014) Mortality after cancer among patients with diabetes mellitus: effect of diabetes duration and treatment. Diabetologia 57:927-934

2. Zakikhani M, Dowling R, Fantus IG, Sonenberg N, Pollak M (2006) Metformin is an AMP kinase-dependent growth inhibitor for breast cancer cells. Cancer Res 66:10269-10273

3. Currie CJ, Poole CD, Gale EAM (2009) The influence of glucoselowering therapies on cancer risk in type 2 diabetes. Diabetologia 52: 1766-1777 